\title{
Theoretical derivation of EMOTION-I model for emotional feel of sensation David Tam
}

Address: Department of Biological Sciences, University of North Texas, Denton, TX 76203, USA

Email: David Tam - dtam@unt.edu

from Seventeenth Annual Computational Neuroscience Meeting: CNS*2008

Portland, OR, USA. 19-24 July 2008

Published: II July 2008

BMC Neuroscience 2008, 9(SuppI I):P79 doi:I0.1 I86/I47I-2202-9-SI-P79

This abstract is available from: http://www.biomedcentral.com/I47I-2202/9/SI/P79

(C) 2008 Tam; licensee BioMed Central Ltd.

The first of a series of emotional models called "Emotional Model of The Theoretical Interpretation Of Neuroprocessing" (EMOTION-I) is derived theoretically to address the origin of emotions in evolutionary process. It is based on first principles without any a priori assumptions about what emotions are and what they are used for. The model is derived based on the necessary principles essential for the survival of an autonomous system (either an organism or an autonomous robot) when interacting with the real-world environment. The contextual abstraction of sensory information provides the basis for the evolution of the emotional context of sensation, i.e., the "feel" in sensation. The emergency of the contextual feel in sensation within the context of the survivability of an autonomous system is derived from the biological principles using reinforcement associative learning to acquire the contextual representation of the external world. The principles of the criteria for hardwiring in reflexes are also derived, which allows for consolidation of the acquired knowledge with respect to the appropriate interaction between the autonomous system and the external world. This theoretical mechanism provides the foundation for the formation of innate ability to recognize pleasant and unpleasant sensation in autonomous systems [1]. This EMOTION-I model forms the pre-processor for the EMOTION-II model for deriving the happy and unhappy emotions.

\section{References}

I. Tam D: EMOTION-I Model: A Biologically-Based Theoretical Framework for Deriving Emotional Context of Sensation in Autonomous Control Systems. The Open Cybernetics \& Systemics Journal 2007, 1:28-46 [http://]. 